\title{
Politische Führung in der Demokratie: Möglichkeiten und Grenzen der vergleichenden Forschung
}

\section{Einleitung}

»Politische Führung « polarisiert. Das gilt offensichtlich nicht nur für die Politik selbst, in der sich vieles um die Erringung und Verteidigung des Anspruchs auf politische Führung und deren Ergebnisse dreht, sondern auch für die wissenschaftliche Auseinandersetzung mit ihr. In der Politikwissenschaft ist der Stellenwert von politischer Führung, von »leadership«, für die liberale Demokratie besonders in normativer Hinsicht heftig umstritten. Einige Autoren erachten politische Führung als unverzichtbar für das Gelingen der Demokratie und sehen die Wissenschaft geradezu in der Pflicht, ihre Einsichten in die Bedingungen einer erfolgreichen Realisierung politischer Führungsansprüche an regierende Eliten weiterzugeben. ${ }^{1}$ Andere hingegen betrachten vor allem Ausprägungen von »transforming leadership« (Burns), von »heroischen «Varianten politischer Führung, als im Kern unvereinbar mit den Grundprinzipien der liberalen Demokratie als einer repräsentativdemokratisch modifizierten Form von Volksherrschaft. ${ }^{2}$

Das außergewöhnlich große politische und demokratietheoretische Konfliktpotential, das sich um politische Führung rankt, hätte diese zu einem besonders intensiv bearbeiten Gegenstand der Politikwissenschaft machen können. Von bedeutenden Einzelleistungen abgesehen, entsprach jedoch lange Zeit nur die amerikanische, primär auf den US-Präsidenten konzentrierte »leadership«-Forschung dieser Erwartung. Erst seit wenigen Jahren ist auch in den deutschsprachigen Ländern ein deutlicher Aufschwung zu beobachten. Er zeigt sich nicht nur auf der Ebene einschlägiger Fachpublikationen, sondern auch in der Gründung spezieller Forschungsbereiche im universitären und außeruniversitären Bereich. $^{3}$

1 Vgl. statt vieler Carnes Lord, The Modern Prince: What Leaders Need to Know Now, New Haven/London 2003; Andrew Rudalevige, » Therefore, Get Wisdomء: What Should the President Know, and How Can He Know It?« in: Governance 22 (2009), S. 177-187.

2 Vgl. Anton Pelinka, »Kritische Hinterfragung eines Konzepts - demokratietheoretische Anmerkungen « in: Annette Zimmer / Regina Maria Jankowitsch (Hg.), Political Leadership, Berlin u.a. 2008, S. 43-67, hier S. 48.

3 Erwähnt seien für Deutschland die Forschungsgruppe Regieren und die NRW School of Governance an der Universität Duisburg-Essen unter der Leitung von Karl-Rudolf Korte sowie das Forschungsprojekt »Leistungsbezogene politische Führung « der Bertelsmann-Stiftung, Gütersloh. In Österreich kam es 2007 zur Begründung einer neuen Sektion der Österreichischen Gesellschaft für Politikwissenschaft, »Political Leadership«.

ZfP 56. Jg. 4/2009 
Das gestiegene wissenschaftliche Interesse an politischer Führung reflektiert seinerseits zu nicht geringen Teilen den Bedeutungsgewinn, den das Sujet während der vergangenen Jahre in der öffentlichen Diskussion über Politik und Demokratie erfahren hat. Das gilt sowohl für die Ebene von »policy leadership« (der inhaltlichen, politisch-materiellen Dimension des politischen Entscheidungsprozesses) als auch für jene von »political leadership « (der prozessualen Dimension politischer Führung). Speist sich der Ruf nach mehr und besserer »policy leadership« durch die Inhaber politischer Führungsämter gegenwärtig primär aus den Folgen einer globalen Finanz- und Wirtschaftskrise, so kann als die wichtigste Ursache der gewachsenen öffentlichen Konzentration auf das Gebaren einzelner Amtsinhaber eine strukturell veränderte Medienberichterstattung über Politik gelten. ${ }^{4}$

Der bemerkenswerte Aufschwung der »leadership «-Forschung bietet Anlass, um nach den potentiellen Leistungen, nach den Möglichkeiten und Grenzen der politikwissenschaftlichen Analyse von politischer Führung zu fragen, insbesondere in ihrem international vergleichenden Zuschnitt. Dabei geht es hier zum einen um die im engeren Sinne wissenschaftlichen Erkenntnispotentiale der vergleichenden »leadership«-Forschung, zum anderen um deren möglichen »gesellschaftlichen Mehrwert«. Die Berücksichtigung dieses zweiten Aspekts ist Ausdruck des Bekenntnisses zu einer Politikwissenschaft, die ein Mindestmaß an gesellschaftlicher Relevanz als Teilbedingung ihrer akademischen Existenzberechtigung begreift. Die Einlösung dieser Forderung muss nicht zwingend in Form einer gezielten Politik- oder Gesellschaftsberatung erfolgen noch ist sie allein einer sich explizit als praxisorientierte »Politikwissenschaft « verstehenden Richtung vorbehalten. ${ }^{5}$ Aber der Stellenwert der Politikwissenschaft in der Demokratie wird ein gutes Stück weit auch daran zu messen sein, ob ihre Befunde auf die eine oder andere Weise geeignet sind, das gesellschaftliche Verständnis der Voraussetzungen und Bedingungen liberaler Demokratie zu erweitern bzw. zu vertiefen.

Aus stilistischen Gründen wird der Begriff der politischen Führung im Weiteren gelegentlich durch »political leadership« ersetzt. Das entspricht der mittlerweile auch in der deutschsprachigen Literatur gängigen Praxis und erleichtert zudem eine Reihe weiterer sprachlicher Differenzierungen. Das speziellere Interesse der nachfolgenden Betrachtungen gilt dem Bereich von »executive leadership« - politischer Führung durch den Regierungschef - mit Konzentration auf die konsolidierten liberalen Demokratien Westeuropas und der USA. Diese thematische Eingrenzung, die Fokussierung auf einen kleinen Kreis von Inhabern politischer Spitzenämter erlaubt es, die aufwendigen Diskussionen über den Zusammenhang von formaler Machtposition und sozialer Füh-

4 Dem Personalisierungsinteresse der Medien kommt dabei die tatsächliche Aufwertung der Inhaber exekutiver Spitzenämter zugute, die im Innenbereich der Exekutive vor allem etwas mit dem erhöhten Koordinierungsbedarf sektoralisierter Politiken, im Außenbereich viel mit der Herausbildung eines Systems internationaler Gipfeltreffen zu tun hat. Vgl. Ludger Helms, Presidents, Prime Ministers and Chancellors: Executive Leadership in Western Democracies, London/New York 2005, S. 6-7.

5 Vgl. hierzu die unterschiedlichen Positionen in Peter Haungs (Hg.), Wissenschaft, Theorie und Philosophie der Politik. Konzepte und Probleme, Baden-Baden 1990. 
rungsrolle, die in anderen, weniger institutionalisierten Kontexten zwingend zu führen sind, weitgehend außer Acht zu lassen. Unverzichtbar ist jedoch eine nähere Bestimmung der zentralen Aspekte von »executive leadership«. Dazu gehört zunächst die Einsicht, dass es bei »executive leadership « keineswegs ausschließlich um das Dirigieren der Exekutive geht, sondern zugleich um exekutivbasierte politische Führung auf der Ebene des politischen Systems. Zu den konstitutiven und Arenen übergreifenden Elementen von »executive leadership « als spezifische Ausprägung von »political leadership« zählen: die Identifikation von gesamtgesellschaftlich relevanten Problemen, die Formulierung und öffentliche Begründung politischer Lösungsvorschläge sowie die für die Realisierung angestrebter Problemlösungen erforderliche Organisation politischer Mehrheiten.

Im Rahmen umfassenderer Führungskonzepte werden häufig Machtgewinn und Machterhalt als weitere zentrale Aspekte von politischer Führung genannt. ${ }^{6}$ Das erscheint angemessen, solange darunter nicht lediglich der Gewinn von Wahlen und der daraus folgende Anspruch auf die Besetzung von Regierungsämtern verstanden werden, denn diese beiden Schritte verdienten eher, als zentrale Bedingungen der Möglichkeit von »executive leadership « klassifiziert zu werden. Sinnvoll, dann jedoch zugleich beinahe selbstverständlich, wäre im Kontext von »executive leadership « ein Verständnis von Machtgewinn und Machterhalt, das auf die Transformation von "political authority « in "political power « bezogen ist. ${ }^{7}$

Ausgehend von der Frage nach dem möglichen Beitrag des politikwissenschaftlichen Studiums von politischer Führung zum Gelingen der repräsentativen Demokratie werden im Weiteren zunächst die Potentiale der politikwissenschaftlich vergleichenden »leadership«-Forschung, anschließend einige ihrer spezifischen Probleme behandelt. Die Schlussbetrachtung wendet sich der Debatte über mögliche Bewertungskriterien politischer Führung zu und beleuchtet die Chancen der politikwissenschaftlichen Forschung, mit ihren Einsichten über den akademischen Bereich hinaus zu strahlen und zu einer gesellschaftsrelevanten Ressource zu werden.

\section{Der mögliche Beitrag von »Comparative Political Leadership» zum Gelingen der repräsentativen Demokratie}

Die Möglichkeiten der politikwissenschaftlichen Forschung über politische Führung, einen Beitrag zur »Demokratisierung der Demokratie ${ }^{8}{ }^{z} \mathrm{zu}$ leisten, sind vielfältig. In einer großen Tradition stehen normativ orientierte Diskussionen über Standards guter politi-

6 Vgl. etwa Nico Grasselt / Karl-Rudolf Korte, Führung in Politik und Wirtschaft. Instrumente, Stile und Techniken, Wiesbaden 2007, S. 54-58.

7 Vgl. Colin Seymour-Ure, Prime Ministers and the Media: Issues of Power and Control, Oxford 2003, S. 51-65.

8 Claus Offe (Hg.), Demokratisierung der Demokratie: Diagnosen und Reformvorschläge, Frankfurt/New York 2003.

ZfP 56. Jg. 4/2009 
scher Führung, die bewusst um die Entwicklung auch moralischer Bezüge bemüht sind. ${ }^{9}$ Größeren Raum nehmen stärker empirisch ausgerichtete exemplarische Darstellungen und Analysen besonders erfolgreicher Führung ein, ${ }^{10}$ die politischen Amtsinhabern und Bürgern Orientierung zu vermitteln vermögen. Sie können sinnvoll ergänzt werden durch Analysen schlechter politischer Führung, ${ }^{11}$ denen eine ähnliche Referenzfunktion zukommen kann. Einen eigenen Bereich markieren Arbeiten zu den institutionellen und organisatorischen Grundlagen politischer Führung; er erstreckt sich von Fragen der Verfassungsreform bis hin zu Aspekten der institutionell-organisatorischen Ausgestaltung und funktionalen Optimierung von Entscheidungsstrukturen. ${ }^{12}$ Hinzu kommen Analysen von Elitenrekrutierungsmustern und Elitenprofilen, die ebenfalls normativ und/oder empirisch angelegt sein können. ${ }^{13}$

Der Fokus der nachfolgenden Betrachtungen liegt auf einem spezielleren Aspekt. Bislang wurde kaum zur Kenntnis genommen, dass die vergleichende »leadership«-Forschung eine besondere Affinität zur Struktur des demokratischen Prozesses in der kompetitiven Arena aufweist. Der Theorie repräsentativer Herrschaft zufolge beruht die Entscheidung bei Wahlen als der zentralen demokratischen Methode auf einer vergleichenden Bewertung unterschiedlicher wahlwerbender Gruppierungen, ihrer Programme und Kandidaten durch die Wähler. Die vergleichende Bilanzierung gezeigter Leistungen und die Abschätzung potentieller künftiger Leistungen politischer Parteien und ihrer Repräsentanten bilden die Grundlage einer rationalen Wahlentscheidung. ${ }^{14}$ Wahlent-

9 Vgl. etwa Moorhead Kennedy / R. Gordon Hoxie / Brenda Repland (Hg.), The Moral Authority of Government, New Brunswick/London 2000; Peter Rinderle, »Welche moralischen Tugenden braucht der Politiker in der liberalen Demokratie? « in: Zeitschrift für Politik 50 (2003), S. 397-422.

10 Vgl. etwa Keith Dean Simonton, Greatness: Who Makes History and Why?, New York 1994; James MacGregor Burns, Transforming Leadership: The New Pursuit of Happiness, London 2003.

11 Vgl. etwa Richard M. Pious, Why Presidents Fail, Lanham, MD 2008; Philip Abbott, "Two Bad Kings and Two Bad Presidents « in: Presidential Studies Quarterly 39 (2009), S. 210-225.

12 Vgl. etwa Hermann Hill, Modernizing Government in Europe, Baden-Baden 2007; KlausDieter Wolf (Hg.), Staat und Gesellschaft - fähig zur Reform? 23. wissenschaftlicher Kongress der Deutschen Vereinigung für Politische Wissenschaft, Baden-Baden 2007; Jerri Killian / Niklas Eklund (Hg.), Handbook of Administrative Reforms: An International Perspective, London u.a. 2008.

13 Vgl. etwa Mattei Dogan (Hg.), Pathways to Power: Selecting Rulers in Pluralist Democracies, Boulder, CO 1989; Jean Blondel / Jean Louis-Thiébault (Hg.), The Profession of Government Minister in Western Europe, London 1991; James D. Davis, Leadership Selection in Six Western Democracies, Westport, CT, 1998; Jean Blondel / Jean Louis-Thiébault (Hg.), The Profession of Government Minister in Western Europe, London 1991; Maurizio Cotta / Heinrich Best (Hg.), Democratic Representation in Europe: Diversity, Change, Convergence, Oxford 2007.

14 In der jüngeren Literatur wird dabei zwischen »representation from below « und »representation from above« unterschieden. Aus letzterer Perspektive betrachtet, stehen die Handlungen der mit politischen Führungsämtern betrauten Akteure im Zentrum der Beziehung zwischen Wählern und Repräsentanten. »In representation from above, the representatives are assigned a more active role. The process of political representation starts with the representatives, who enter the political process with their views and put these views to the citizens for their approval.« So Rudy B. Andeweg / Jacques J. A. Thomassen, »Modes of Political Representation: 
scheidungen werden aber im Kontext generellerer Erwartungen an politische Führung getroffen, hinter denen sowohl die Leistungen und Angebote der Regierung als auch jene der Opposition zurückbleiben können. Zum Teil sind diese enttäuschten Erwartungen das Ergebnis populistischer Strategien und Szenarien politischer Akteure, die sich davon den Gewinn der Wählergunst versprechen - gerade dann, wenn sie keine Regierungsverantwortung tragen bzw. diese nur bedingt anstreben. ${ }^{15}$

Eine der zentralen Thesen dieser Abhandlung lautet jedoch, dass nicht sämtliche der enttäuschten Erwartungen an politische Führung »hausgemacht sind. Die verbreitete »Erwartungslücke «, ${ }^{16}$ mit der sich Regierungen und ihre Protagonisten konfrontiert sehen, hat auch etwas mit der Anlegung fragwürdiger, unreflektiert aus anderen politischen und institutionellen Kontexten importierten Bewertungsmaßstäben zu tun. Die Etablierung eines Systems internationaler Gipfeltreffen, auf denen die Regierungschefs der unterschiedlichsten Länder unter regem Interesse der Medien regelmäßig zusammenkommen, begünstigt die Vorstellung, dass die beteiligten Akteure über grundsätzlich ähnliche Handlungsbedingungen und -ressourcen verfügen. Angereichert werden entsprechende Vermutungen durch speziellere Leitnormen politischer Führung. Nicht nur in Deutschland wurde vor allem den beiden angelsächsischen Modelldemokratien USA und Großbritannien stets eine zentrale Referenzfunktion zuerkannt, ${ }^{17}$ oftmals mit einer problematischen »Verdichtung « unterschiedlicher Aspekte zur besten aller Welten. Zu den zentralen Komponenten »guter politischer Führung « werden dabei parteienübergreifende Autorität und Integrationskraft (wie sie potentiell vor allem amerikanischen Präsidenten zugeschrieben wird) und eine auf Kooperation und Kompromissbildung kaum angewiesene Durchsetzungsfähigkeit des Regierungschefs (als vermeintliches Merkmal der britischen Westminster-Demokratie) gezählt.

Der Anspruch der vergleichenden »leadership«-Forschung im hier umrissenen Problemkontext muss es sein, Differenzierungen zu erarbeiten, die dabei helfen können, systembedingt unangemessene Erwartungen an politische Führung zu vermeiden. Dabei geht es nicht um eine Erziehung zur Bescheidenheit. Die Einsicht in das unter bestimmten Bedingungen Mögliche schafft vielmehr gleichermaßen die Voraussetzungen für eine

Toward a New Typology « in: Legislative Studies Quarterly 30 (2005), S. 507-528, hier 511. Die Betonung der zentralen Bedeutung politischer Führung ist freilich kein Merkmal der jüngeren Parlamentarismusliteratur; sie kennzeichnete vielmehr bereits die Sichtweise Walter Bagehots, dem historischen Begründer der so genannten »realistischen « Position; vgl. Eberhard SchüttWetschky, »Haben wir eine akzeptable Parlamentarismustheorie? « in: Jürgen Hartmann / Uwe Thaysen (Hg.), Pluralismus und Parlamentarismus in Theorie und Praxis, Opladen 1992, S. 91-112, hier $95 \mathrm{f}$.

15 Vgl. Pelinka, Kritische Hinterfragung eines Konzepts, aaO. (FN 2), S. 2.

16 Vgl. Arvind Raichur / Richard W. Waterman, »The Presidency, the Public, and the Expectations Gap« in: Richard W. Waterman (Hg.), The Presidency Reconsidered, Ithaca, IL 1993, S. 1-21.

17 Seit dem Amtsantritt von Präsident Barack Obama Anfang 2009 sind die Augen der Weltöffentlichkeit freilich vor allem auf diesen gerichtet, während die internationale Ausstrahlungswirkung der britischen "premiership « nach dem Ausscheiden Tony Blairs deutlich nachgelassen hat. Als Referenzmodelle bleiben jedoch beide Systeme, unabhängig von aktuellen personalpolitischen Konstellationen, von zentraler Bedeutung. 
positive Wertschätzung des Erreichten wie für eine substantielle Kritik greifbarer Defizite.

\section{Möglichkeiten der vergleichenden »leadership«-Forschung}

Die Ausgangsthese dieses Abschnitts lautet: Institutionen bilden nach wie vor den aussichtsreichsten Ausgangspunkt für eine Bestimmung der strukturellen Rahmenbedingungen und Spielräume politischer Führung. ${ }^{18}$ Freilich greift eine Konzentration auf die formalen, verfassungsrechtlichen Regeln, wie sie Arbeiten aus der Richtung des klassischen Institutionalismus kennzeichnete, zu kurz. Gerade das Studium politischer Führung und ihrer Strukturbedingungen kommt nicht ohne die Einbeziehung »informaler Institutionen «, kurz: ungeschriebener Regeln, aus. ${ }^{19}$ Die zumeist größere Stabilität formaler Institutionen und ihr normativ herausgehobener Status sprechen jedoch dafür, grundlegende Typologien von »leadership«-Umwelten an den formalen institutionellen Arrangements, insbesondere den jeweiligen Verfassungsregeln, festzumachen.

Die erste im Rahmen einer Vermessung des institutionellen Handlungsrahmens politischer Führung zu berücksichtigende Dimension betrifft die Regierungsform. Mit Blick auf diese wird traditionell zwischen parlamentarischen und präsidentiellen Demokratien unterschieden ${ }^{20}$ - eine im Kern auf das Verhältnis zwischen Exekutive und Legislative konzentrierte Differenzierung, die jedoch erheblich weiterreichende Implikationen besitzt. In der Familie der konsolidierten liberalen Demokratien verkörpern die Vereinigten Staaten nach wie vor die einzige echte präsidentielle Demokratie, während die politische Landkarte Westeuropas ganz eindeutig von parlamentarischen Demokratien beherrscht wird.

18 In konzeptueller Hinsicht bewährt hat sich der Ansatz des »akteurzentrierten Institutionalismus «; vgl. Renate Mayntz / Fritz W. Scharpf, »Der Ansatz des akteurzentrierten Institutionalismus « in: dies. (Hg.), Gesellschaftliche Selbstregelung und politische Stenerung, Frankfurt a.M./New York 1995, S. 39-72; Fritz W. Scharpf, Interaktionsformen: Akteurzentrierter Institutionalismus in der Politikforschung, Opladen 2000. Von ihm sind auch die nachfolgenden Ausführungen angeleitet. Dabei werden Institutionen als Regeln bzw. Regelsysteme begriffen, die das Handeln individueller wie korporativer Akteure einerseits ermöglichen, andererseits begrenzen.

19 Vgl. grundlegend Gretchen Helmke / Steven Levitsky, Informal Institutions and Comparative Politics: A Research Agenda in: Perspectives on Politics 2 (2004), S. 725-740; zum Topos des Informalen bzw. der Informalisierung im spezielleren Kontext der »leadership «-Forschung am Beispiel der Bundesrepublik Ludger Helms, »Die Informalisierung des Regierungshandelns in der Bundesrepublik « in: Zeitschrift für Staats- und Europawissenschaften 3 (2005), S. 70-96; Wolfgang Rudzio, Informelles Regieren, Wiesbaden 2005. - Auch und gerade die historische Herausbildung von Strukturen politischer Führung bleibt ohne die Einbeziehung informaler Institutionen unverständlich. Vgl. hierzu Wolfgang Reinhard, Geschichte der Staatsgewalt. Eine vergleichende Verfassungsgeschichte Europas von den Anfängen bis zur Gegenwart, München 2000, S. 141-196.

20 Vgl. Winfried Steffani, »Zur Unterscheidung parlamentarischer und präsidentieller Regierungssysteme « in: Zeitschrift für Parlamentsfragen 14 (1983), S. 391-401; Arend Lijphart (Hg.), Parliamentary versus Presidential Government, Oxford 1992. 
Gravierende Unterschiede der beiden Regierungsformen mit Blick auf den Handlungsspielraum des »chief executive « betreffen zunächst das Innenleben der Exekutive. Dem ganz auf den Präsidenten hin konzentrierten Konzept politischer Verantwortlichkeit entsprechend ist die Entscheidungsautonomie amerikanischer Präsidenten innerhalb der Exekutive, mit wenigen Einschränkungen, potentiell grenzenlos - nicht nur bei der Organisation der so genannten »presidential branch «, jener erst in den dreißiger Jahren des 20. Jahrhunderts geschaffenen speziellen organisatorisch-personellen »SupportStruktur « des Präsidenten. Das amerikanische Kabinett ist kein Kollegialorgan, dem der Präsident ein Mehrheitsvotum abzuringen hätte, sondern eine Versammlung ohne verfassungsrechtlichen Status, deren einzelne Mitglieder eine weitgehende politische Abhängigkeit vom Weißen Haus verbindet. ${ }^{21}$ Kein Regierungschef Westeuropas verfügt über vergleichbar weit reichende Gestaltungsspielräume im Bereich der Exekutive. Das gilt selbst, und in mancher Hinsicht gerade, für britische Premiers. ${ }^{22}$ Regierungschefs parlamentarischer Demokratien müssen um eine substantiell einflussreiche Position innerhalb eines zunehmend komplexer strukturierten Exekutivterritoriums grundsätzlich ringen. Dabei sind die jeweiligen »Startbedingungen « europäischer Premiers freilich sehr unterschiedlich: Als besonders mächtig gelten mit Blick auf ihre strukturelle Position innerhalb der Exekutive die Regierungschefs von Ländern wie Großbritannien, Irland, Deutschland, Spanien und Griechenland, als traditionell eher schwach jene von Ländern wie Norwegen oder Italien. ${ }^{23}$

Ein zweiter, im hier gegebenen Kontext zentraler Unterschied zwischen beiden Regierungsformen bezieht sich auf die Struktur der Exekutivspitze: Während in parlamentarischen Systemen die Ämter des Regierungschefs und Staatsoberhaupts institutionell und personell voneinander getrennt sind, vereinigen Präsidenten präsidentieller Systeme beide Ämter in ihren Händen. Daraus ergeben sich gravierend unterschiedliche Handlungsbedingungen: Ceteris paribus sind die strukturellen Chancen parlamentarischer Regierungschefs zu einer nationalen, gleichsam »überparteilichen « Integrationsinstanz zu werden, bedeutend geringer als jene von Präsidenten präsidentieller Systeme. Zumindest wichtige Teile der Integrationsfunktion bleiben in der parlamentarischen Demokratie üblicherweise dem Staatsoberhaupt vorbehalten. »Quasi-präsidiale « Autorität und damit einhergehende erweiterte politische Gestaltungsmacht erlangen Regierungschefs parlamentarischer Demokratien nur in seltenen Ausnahmesituationen, am ehesten als

21 Vgl. Ludger Helms, »Die historische Entwicklung und politische Bedeutung des Kabinetts im Regierungssystem der USA « in: Politische Vierteljabresschrift 40 (1999), S. 65-92.

22 Vgl. Richard E. Neustadt, »White House and Whitehall« in: Anthony King (Hg.), The British Prime Minister, London 1969, S. 131-147.

23 Vgl. Anthony King, "Chief Executives in Western Europe« in: Ian Budge / David McKay (Hg.), Developing Democracy. Comparative Research in Honour of J.F.P. Blondel, London 1994, S. 150-163; Eoin O’Malley, »The Power of Prime Ministers: Results of an Expert Survey « in: International Political Science Review 28 (2007), S. 1-27. 
»Krisenmanager« in Phasen innerer und/oder äußerer Bedrohung des Gemeinwesens. ${ }^{24}$ Die strukturelle Integration der Ämter des Regierungschefs und Staatsoberhaupts im Präsidentialismus muss sich jedoch nicht zwingend als vorteilhaft erweisen. Die spezifische Struktur des amerikanischen Präsidentenamtes generiert kaum zu überbietende Erwartungen an die Integrations- und Repräsentationsleistung des Amtsinhabers, die alles andere als leicht zu befriedigen sind. Im Falle enttäuschter Hoffnungen können diese zu einer schweren Hypothek werden, die den Führungsanspruch von Präsidenten beschädigen und deren politischen Durchsetzungsvermögen verringern. ${ }^{25}$ Aus dieser Perspektive betrachtet werden scheinbar paradoxe Befunde der jüngeren Forschung verständlich, die auf einen positiven Einfluss restriktiver Bedingungen politischer Führung, wie insbesondere den Zustand von »divided government «, auf die politische Performanz amerikanischer Präsidenten deuten; sie sind als Katalysatoren einer willkommenen »Erwartungsentlastung zu betrachten. $^{26}$

Die auf der Ebene des politischen Prozesses insgesamt schwerwiegendsten Unterschiede zwischen parlamentarischen und präsidentiellen Systemen entspringen jedoch dem Prinzip parlamentarischer Verantwortlichkeit der Regierung, aus dem sich weitere Strukturcharakteristika des Parlamentarismus ergeben, darunter insbesondere die spezifische Gewaltenfusion zwischen Regierung und parlamentarischer Mehrheit und der institutionalisierte Gegensatz von Regierungsmehrheit und parlamentarischer Opposition. Die Existenz eines funktionalen Handlungsverbunds zwischen Regierung und Parlamentsmehrheit im Parlamentarismus sorgt nicht nur für eine, gemessen an amerikanischen Verhältnissen, strikte Parteidisziplin innerhalb und außerhalb der parlamentarischen Arena. Sie ist ferner dafür verantwortlich, dass - aus zeitlicher Perspektive betrachtet - der entscheidende Teil der politischen Führungsleistung durch den Regie-

24 Vgl. Arjen Boin / Paul 't Hart / Eric Stern / Bengt Sundelius, The Politics of Crisis Management. Public Leadership under Pressure, Cambridge 2005; Arjen Boin / Allan McConnel / Paul 't Hart (Hg.), Governing after Crisis: The Politics of Investigation, Accountability and Learning, Cambridge 2008.

25 Als wichtigstes jüngeres Beispiel hierfür kann die Präsidentschaft George W. Bushs gelten, der - entgegen anders lautender Ankündigungen - früh das Profil eines »partisan president « mit entsprechend geringem Integrationspotential entwickelte, das sich mit fortdauernder Amtszeit zunehmend auch als Restriktion auf der Ebene der Entscheidungspolitik niederschlug. Vgl. Bert A. Rockman, »Presidential Leadership in an Era of Party Polarization - The George W. Bush Presidency « in: Colin Campbell / Bert A. Rockman (Hg.), The George W. Bush Presidency. Appraisals and Prospects, Washington, DC 2004, S. 319-357, hier S. 349 f.; Richard M. Skinner, »George W. Bush and the Partisan Presidency in: Political Science Quarterly 123 (2008/9), No. 4, S. 605-622. - Für die parlamentarischen Demokratien gilt umgekehrt, dass unter bestimmten Umständen gerade die effektive Ausfüllung der Repräsentativ- und Integrationsfunktion durch das Staatsoberhaupt dem Regierungschef zugutekommen kann wie mit Blick auf die Bundesrepublik für das Gespann von Weizsäcker/Kohl gemutmaßt wurde.Vgl. Ludger Helms, Regierungsorganisation und politische Führung in Deutschland, Wiesbaden 2005, S. 167.

26 »Divided government « erscheint dabei als »an escape hatch for presidents, allowing them to maintain the appearance of heroism without needing to deliver the goods «; so Michael E. Bailey, »The Heroic Presidency in the Era of Divided Government« in: Perspectives on Political Science 31 (2002), No. 1, S. 35-45, hier S. 44. 
rungschef im Vorfeld der Eröffnung des legislativen Verfahrens im engeren Sinne zu erbringen ist. Hat eine geplante Maßnahme erst einmal die Zustimmung des Kabinetts erhalten, bleibt für den Regierungschef in aller Regel wenig mehr zu tun. Ausnahmen betreffen am ehesten komplexe föderative Systeme, in denen regelmäßig um die politische Unterstützung weiterer Akteure gerungen werden muss.

Etwas ganz anderes gilt für die präsidentielle Demokratie: Formal nicht einmal mit einem legislativen Initiativrecht ausgestattet, beginnt der eigentliche Kampf des Präsidenten um eine Vorlage in aller Regel erst nach der Eröffnung des legislativen Verfahrens - mit selbst in Phasen von »unified government « (Präsidentenamt und Kongress umspannenden Mehrheiten einer Partei) ungewissem Ausgang. Dabei bedienen sich Präsidenten aller erdenklichen Instrumente und Strategien, darunter jene des »going public«: aufwendige öffentliche Mobilisierungskampagnen, deren eigentlicher Adressat weniger die amerikanische Bevölkerung als die Washingtoner Legislativelite ist. ${ }^{27}$ Der bedeutend höhere Grad an Öffentlichkeit politischer Führung im Präsidentialismus gilt amerikanischen Beobachtern als zentrales Element der politischen Verwundbarkeit von Präsidenten ${ }^{28}$ - ein wichtiger Hinweis mit einiger Relevanz auch für die Bewertung der vielfach konstatierten »Präsidentialisierung « politischer Führung in parlamentarischen Demokratien, die den meisten Betrachtern als Ursache und Ausdruck einer strukturellen Stärkung von Regierungschefs erscheint. ${ }^{29}$

$\mathrm{Zu}$ den maßgeblichen Unterschieden in den strukturellen Ausgangsbedingungen politischer Führung im Präsidentialismus und im Parlamentarismus gehört ferner das gravierend unterschiedliche Gewicht politischer Parteien für die Realisierung des Führungsanspruchs des jeweiligen »chief executive«. Anders als es die außerordentlich harten innerparteilichen Auseinandersetzungen über die Auswahl des Präsidentschaftskandidaten vermuten lassen würden, ist die Unterstützung eines amtierenden Präsidenten durch seine Partei geradezu selbstverständlich. Die zeit- und kraftraubenden Versuche von Regierungschefs parlamentarischer Regierungssysteme, den programmatischen Kurs der eigenen Partei im gewünschten Sinne zu steuern oder jedenfalls zu beeinflussen,

27 Vgl. Samuel Kernell, Going Public: New Strategies of Presidential Leadership, 4. Aufl., Washington, DC 2006.

28 Vgl. Bert A. Rockman, »The American Presidency in Comparative Perspective: Systems, Situations, and Leaders" in: Michael Nelson (Hg.), The Presidency and the Political System, 7. Aufl., Washington, DC 2003, S. 48-75, hier S. 56.

29 Gelegentlich wird jedoch auch diese Dimension von »Präsidentialisierung « erkannt; so bei Paul Webb / Thomas Poguntke, »The Presidentialization of Contemporary Democratic Politics: Evidence, Causes, and Consequences « in: Thomas Poguntke / Paul Webb (Hg.), The Presidentialization of Politics. A Comparative Study of Modern Democracies, Oxford 2005, S. 336-356, hier S. 353. Angesichts des Verzichts parlamentarischer Regime auf feste Amtszeiten des Regierungschefs ist die politische Verwundbarkeit des »chief executive« durch »Präsidentialisierung « im Sinne einer tendenziellen Auflösung eines parteienbasierten »leadership «Anspruchs in parlamentarischen Demokratien potentiell sogar bedeutend größer als im Präsidentialismus selbst. Vgl. zu den Effekten der »Präsidentialisierung « mit Blick auf den Verfall politischer Macht am Beispiel Tony Blairs, Michael Foley, »The Presidential Dynamics of Leadership Decline in Contemporary British Politics: The Illustrative Case of Tony Blair « in: Contemporary Politics 14 (2008), S. 53-69. 
sind in den USA praktisch unbekannt. Zum »party government « amerikanischer Prägung gehört vielmehr eine gleichsam »natürliche«, von niemandem ernsthaft in Frage gestellte Direktionsfunktion des Präsidenten. ${ }^{30}$ Eine vergleichbar weitreichende Beherrschung der Regierungspartei von Premierministern parlamentarischer Demokratien zu fordern, wäre »fair « noch realistisch.

Diese und weitere Vorbehalte in Bezug auf die Vergleichbarkeit der strukturellen Handlungsbedingungen von »chief executives « im Parlamentarismus und Präsidentialismus gelten auch für einen Vergleich parlamentarischer Regierungschefs mit Präsidenten »semi-präsidentieller « Systeme. Der Prototyp der »semi-präsidentiellen« Demokratie, die sich im vorherrschenden Verständnis der Regierungslehre durch eine Kombination der Strukturmerkmale parlamentarischer und präsidentieller Systeme auszeichnet, ${ }^{31}$ bleibt in Westeuropa die V. Republik Frankreich. Die heute zunehmend befürwortete Klassifikation »semi-präsidentieller« Systeme als Subtypus der parlamentarischen Demokratie erscheint vor allem mit Blick auf Phasen geteilter parteipolitischer Kontrolle von Präsidentenamt und Nationalversammlung angemessen. In ihnen gibt der Premierminister, nicht der Präsident, den Ton an. In Phasen einheitlicher Mehrheitsverhältnisse eröffnen »semi-präsidentielle« Systeme dem Präsidenten hingegen oftmals außergewöhnlich großzügige Handlungs- und Gestaltungsspielräume, die über jene von Premierministern parlamentarischer Demokratien wie von Präsidenten präsidentieller Demokratien weit hinausreichen. Die Bezeichnung »Superpräsidentialismus « erscheint deshalb keineswegs ausschließlich auf Phasen der russischen Politik nach 1990 anwendbar. ${ }^{32}$ Als Referenzakteure für die vergleichende Bewertung der Performanz von Regierungschefs (rein) parlamentarischer Demokratien und vor allem solcher in machtdistributiven Koalitionsdemokratien sind Präsidenten »semi-präsidentieller « Systeme deshalb üblicherweise besonders ungeeignet.

Die Regierungsform ist jedoch nicht die einzige institutionelle Variable von besonderem Gewicht. In Teilen der jüngeren »leadership«-Forschung gilt eine andere institutionelle Dimension von Regierungssystemen - nämlich die Anzabl und Stärke institutioneller "Mitregenten " oder »Vetospieler" - als im Zweifelsfalle noch wichtiger als der

30 Vgl. James W. Davis, The President as Party Leader, Boulder, CO 1992; Michael J. Korzi, A Seat of Popular Leadership: The Presidency, Political Parties, and Democratic Leadership, Amherst 2004.

31 Dazu gehören insbesondere die (Ko-)Existenz von Präsident und Premierminister innerhalb eines Systems mit parlamentarischer Regierungsverantwortung und Direktwahl eines Präsidenten mit fester Amtszeit. Vgl. Robert Elgie, »Semi-Presidentialism: Concepts, Consequences and Contesting Explanations in: Political Studies Review 2 (2004), S. 314-330. Sofern von einem Primärmerkmal der Unterscheidung von parlamentarischen und präsidentiellen Systemen - der parlamentarischen Verantwortlichkeit der Regierung - ausgegangen wird, erscheint das »semi-präsidentielle« System als eine »parlamentarische Demokratie mit Präsidialdominanz bzw. Präsidialhegemonie«, so Steffani, Zur Unterscheidung parlamentarischer und präsidentieller Regierungssysteme, aaO. (FN 20), S. 396.

32 Vgl. Timothy J. Colton, "Superpresidentialism and Russia's Backward State« in: Post-Soviet Affairs 11 (1995), Nr. 2, S. 144-148. Vgl. etwa im französischen Kontext Robert Ponceyri, »La Cinquième République au risque de l'hyperprésidentialisme« in: Revue politique et parlementaire Nr. 109 (2007), S. 176-211. 
Präsidentialismus/Parlamentarismus-Gegensatz. ${ }^{33}$ Tatsächlich bemessen sich die strukturellen Handlungsspielräume von Regierungen nicht unwesentlich nach der Anzahl und Stärke von Akteuren wie zweiten Kammern, Verfassungsgerichten oder unabhängigen Zentralbanken. Die institutionelle Komplexität von Regierungssystemen beeinflusst jedoch nicht nur die politischen Entscheidungsspielräume im Sinne der Handlungsautonomie der Gubernative, sondern auch die Bedingungen öffentlicher politischer Führung und Kommunikation. Das betrifft Vivian Schmidt zufolge insbesondere die Rolle und den Stellenwert koordinativer und kommunikativer Diskurse. ${ }^{34}$ In komplexen Systemen (»compound polities«), wie beispielsweise der Bundesrepublik Deutschland, ist für Schmidt der koordinative Diskurs in hohem Maße elaboriert, der kommunikative Diskurs hingegen üblicherweise dünn - nicht zuletzt deshalb, weil vermieden werden soll, dass die Details von mühsam hinter verschlossenen Türen gefundenen Kompromissen durch eine detaillierte öffentliche Diskussion offen gelegt werden. In institutionell einfach gestrickten, machtkonzentrierenden Systemen (»simple polities«) verhält es sich genau umgekehrt: Dort sorgt gerade die weitgehende Abwesenheit weitreichender Policy-Koordination dafür, dass politische Akteure ein stärkeres Bestreben entwickeln, neue Ideen durch eine intensive Kommunikation mit der breiteren Öffentlichkeit zu legitimieren.

Institutionelle Unterschiede auf dieser Ebene erklären die trotz der gemeinsamen Regierungsform beträchtlichen Unterschiede des Regierens in Ländern wie Deutschland oder Österreich einerseits und Großbritannien andererseits in erheblichem Maße mit. Zugleich erscheinen aus dieser Perspektive die strukturellen Voraussetzungen politischer Führung in Systemen wie der Bundesrepublik und den USA einander nicht so vollständig unähnlich zu sein wie ein streng auf die Parlamentarismus/Präsidentialismus-Dimension beschränkter Vergleich suggeriert. Hier wie dort zwingt die große Anzahl und Stärke institutioneller »Mitregenten « und »Vetospieler« Akteure zur Verfolgung von auf Inklusion und Kompromiss hin orientierten Führungsstrategien.

Gerade diese allgemeinen Ähnlichkeiten können jedoch zu problematischen Bewertungen verleiten. Eine vergleichbar hohe »Vetospielerdichte in zwei Systemen hebt die Wirkung und Funktionslogik unterschiedlicher Regierungsformen nicht auf wie gelegentlich auch mit Blick auf den spezielleren Gegenstand der exekutiven politischen Füh-

33 Vgl. Bert A. Rockman, »The Performance of Presidents and Prime Ministers and of Presidential and Parliamentary Systems « in: Kurt von Mettenheim (Hg.), Presidential Institutions and Democratic Politics: Comparing Regional and National Contexts, Baltimore/London 1997, S. 48-64, hier S. 60; B. Guy Peters, »The Separation of Powers in Parliamentary Systems « in: von Mettenheim (Hg.), Presidential Institutions and Democratic Politics, aaO., S. 67-83, hier S. 72.

34 Vgl. Vivian A. Schmidt, »Democracy in Europe: The Impact of European Integration« in: Perspectives on Politics 3 (2005), S. 761-779, hier S. 773 f. Koordinative Diskurse werden vor allem zwischen Policy-Akteuren (wie Experten, Interessengruppen, Karrierebeamten etc.) geführt, die auf sachpolitische Inhalte bezogene Einigungsprozesse in der Policy-Community koordinieren. Kommunikative Diskurse werden hingegen von politischen Akteuren (Politikern, Wahlkampagnenmanagern etc.) geführt; durch sie werden die im koordinativen Diskurs entwickelten Ideen an die breitere Öffentlichkeit kommuniziert. 
rung in unterschiedlichen Demokratien argumentiert wurde. ${ }^{35}$ Vergleichende Studien über das Regieren unter den Bedingungen des »institutionellen Pluralismus « verdeutlichen, dass nicht nur die Rolle gesellschaftlicher Akteure wie der Interessenverbände, sondern auch die strukturelle Position von Verfassungsorganen wie zweiten Kammern oder Verfassungsgerichten im Parlamentarismus und Präsidentialismus deutlich andere sind. ${ }^{36}$

Die politische Realität, in der die Regierungschefs der gegenwärtigen Demokratien agieren, ist ungleich komplexer als es die Regierungsformenlehre in ihrem Bestreben nach Orientierung vermittelnder Klassifizierung zu erfassen vermöchte. Selbst innerhalb der Gruppe von Systemen, die mit Blick auf die zentralen institutionellen Parameter horizontaler und vertikaler Gewaltenteilung in dieselbe Kategorie fallen, gibt es gravierende strukturelle Unterschiede hinsichtlich der politischen Bedingungen von »executive leadership«.

Als Variable von besonderem Gewicht gilt zu Recht das Regierungsformat, ganz besonders der Unterschied zwischen Einparteienregierungen und Koalitionsregierungen. Unter sonst gleichen Bedingungen ist die Machtposition des Premiers in einer Einparteienregierung eine deutlich stärkere als an der Spitze einer Koalitionsregierung. Das zeigt sich vor allem, aber keineswegs nur bei Personalentscheidungen im Kontext von Regierungs(um)bildungen. ${ }^{37}$ Umfragen unter Ministern westeuropäischer Länder zeigen etwa, dass Premierminister an der Spitze von Einparteienregierungen deutlich intensiver in unterschiedliche Politikfelder involviert sind als Koalitionspremiers; sie verfügten im Urteil der Befragten ferner über eine rund dreimal so große Fähigkeit wie »Koalitionspremiers «, ihren politischen Einfluss innerhalb der Exekutive mit zunehmender Amtszeit zu vergrößern. ${ }^{38}$ Auch Dynamiken funktionaler Informalisierung innerhalb der Exekutive, die sich in allen Regierungssystemen beobachten lassen, gehen im Kontext von Einparteienregierungen seltener auf Kosten des Premiers. Während die in der großen Mehrzahl parlamentarischer Koalitionsdemokratien bekannten Koalitionsrunden und -ausschüsse den Regierungschef entscheidungspolitisch schwächen können, bleiben Kabinettsausschüsse als wichtigstes funktionales Äquivalent einer koalitionspolitischen Informalisierung des Kabinettssystems in Systemen mit Einparteienregierung in der Regel

35 Vgl. Michael Stoiber, »Politische Führung und Vetospieler. Einschränkungen exekutiver Regierungsmacht « in: Everhard Holtmann / Werner J. Patzelt (Hg.), Führen Regierungen tatsächlich? Zur Praxis gouvernementalen Handelns, Wiesbaden 2008, S. 35-57.

36 Vgl. Ludger Helms, "Regieren unter den Bedingungen des institutionellen Pluralismus: ein deutsch-amerikanischer Vergleich « in: Politische Vierteljabresschrift 44 (2003), S. 66-85; Philipp Dann, »The Gubernative in Presidential and Parliamentary Systems: Comparing Organizational Structures of Federal Governments in the USA and Germany « in: Zeitschrift für ausländisches öffentliches Recht und Völkerrecht 66 (2006), S. 1-40.

37 Vgl. John D. Huber / Cecilia Martinez-Gallardo, »Replacing Cabinet Ministers: Patterns of Ministerial Stability in Parliamentary Democracies « in: American Political Science Review 102 (2008), S. 169-180.

38 Vgl. Wolfgang C. Müller / Wilfried Philipp / Peter Gerlich, »Prime Ministers and Cabinet Decision-Making Processes « in: Jean Blondel / Ferdinand Müller-Rommel (Hg.), Governing Together. The Extent and Limits of Collective Decision-Making in Western European National Cabinets, London 1993, S. 223-256, hier S. 232 f. 
auf den Premier konzentriert und können dessen entscheidungspolitisches Gewicht sogar noch vergrößern. Der strukturelle Vorteil, den Einparteienregierungen aus Sicht des Regierungschefs bieten, kann freilich gegebenenfalls durch andere restriktive Faktoren minimiert werden. In Richtung eines funktionalen Äquivalents koalitionspolitischer Strukturen schlägt insbesondere ein hoher interner Faktionalisierungsgrad alleinregierender Parteien. Dennoch behält die »Daumenregel «, nach der Einparteienregierungen den Handlungsspielraum von Regierungschefs strukturell vergrößern, ihre Gültigkeit. Schließlich sind auch Koalitionsregierungen vor dem Problem einer internen Zerklüftung der regierenden Parteien nicht gefeit. Unter dem Stichwort »Regierungsformat « ist jedoch weiter zwischen unterschiedlichen Koalitionsformaten zu differenzieren. In Vielparteienkoalitionen ohne eindeutig dominante Partei ist die Macht des Premiers praktisch zwingend stark eingeschränkt. Daneben bringen vor allem große Koalitionen ungewöhnlich weitreichende Restriktionen des Handlungsspielraums von Regierungschefs mit sich wie (nicht nur) die deutschen Erfahrungen lehren. ${ }^{39}$

Auch die interne Struktur und die Ressourcenausstattung der »Regierungszentralen" (in Deutschland des Kanzleramts) verdient es, zu den maßgeblichen Bestimmungsfaktoren der Handlungs- und Durchsetzungsmächtigkeit von Regierungschefs gezählt zu werden. Auf den Einfluss dieses Faktors weisen nicht zuletzt Arbeiten über strukturell schwache Premiers in jungen Demokratien hin. ${ }^{40}$ Signifikante Unterschiede auf dieser Ebene gibt es freilich auch zwischen den konsolidierten Demokratien Westeuropas. ${ }^{41}$ Mit Blick auf das »monitoring « des Regierungsprozesses haben sich besonders Organisationsstrukturen mit so genannten »Spiegelreferaten « als effektiv erwiesen, die an der jeweiligen Ressortstruktur des Kabinetts ausgerichtet sind. Ansonsten gilt - trotz der vor allem aus den USA bekannten möglichen kontraproduktiven Effekte eines »overstaffing « mit der wachsenden Gefahr internen Wettbewerbs und geteilter Loyalitäten unter den Mitarbeitern ${ }^{42}$ - dass eine großzügige Personalausstattung der Regierungszentrale dem Durchsetzungsanspruch des Regierungschefs in aller Regel zugute kommt. ${ }^{43}$ Die nicht nur aus der Geschichte der Bundesrepublik bekannte Veränderungsdynamik in der Funktionsweise und Leistungsfähigkeit von Regierungszentralen innerhalb eines Sys-

39 Vgl. Karlheinz Niclauß, „Kiesinger und Merkel in der Großen Koalition« in: Aus Politik und Zeitgeschichte B 16/2008, S. 3-10; Michel Fabrequet (Hg.), Les grandes coalitions en Allemagne et en Autriche (Revue d'Allemagne et des Pays de langue allemand 40, Heft 4), Straßburg, 2008; Wolfgang C. Müller / Kaare Strøm (Hg.), Coalition Governments in Western Europe, Oxford 2000.

40 Vgl. im osteuropäischen Kontext etwa Thomas A. Baylis, »Embattled Executives: Prime Ministerial Weakness in East Central Europe«, in: Communist and Post-Communist Studies 40 (2007), 81-106, hier S. 90.

41 Vgl. Ferdinand Müller-Rommel, »Ministers and the Role of the Prime Ministerial Staff « in: Blondel / Müller-Rommel, Governing Together, aaO. (FN 38), S. 131-152.

42 Gelegentlich wurde der stetige Zuwachs an Personal und struktureller Komplexität des White House Office gar als ein wesentliches Moment der Schwäche von US-Präsidenten identifiziert; vgl. Richard E. Neustadt, »The Weakening White House« in: British Journal of Political Science 31 (2001), S. 1-11, hier S. 8 f.

43 Vgl. B. Guy Peters / R. A. W. Rhodes / Vincent Wright (Hg.), Administering the Summit: The Administration of the Core Executive in Developed Countries, London 2000. 
tems deuten darauf hin, dass diese Komponente der Rahmenbedingungen politischer Führung nicht unbeeinflusst ist vom jeweiligen Input des Regierungschefs. ${ }^{44}$

Die parlamentarischen Mebrbeitsverbältnisse zwischen Regierung und Opposition stellen eine dritte Variable von herausragendem Gewicht dar. Unter sonst gleichen Bedingungen können es Minderheitsregierungen und ihre Protagonisten hinsichtlich ihrer strukturellen Gestaltungsspielräume mit Mehrheitsregierungen nicht aufnehmen. Eine Umsetzung legislativer Agenden ist für Minderheitsregierungen ausschließlich auf der Basis weitgehend ungesicherter parlamentarischer Ad-hoc-Mehrheiten möglich. In diesem Sinne wurde etwa dem dänischen Minderheitsparlamentarismus attestiert, hinsichtlich der Beziehungen zwischen Exekutive und Legislative von einer »präsidentiellen Logik « bestimmt zu sein. ${ }^{45}$ Allerdings ist es um die Handlungsspielräume der Regierung bzw. des Regierungschefs keineswegs zwingend umso besser bestellt, je größer die parlamentarische Mehrheitsbasis im Einzelfall ist. Übergroße Mehrheiten scheinen eher die Hemmschwellen von Parlamentariern zu senken, im Zweifelsfalle auch einmal gegen die Regierung zu stimmen. Daraus entstehen nur gelegentlich ernsthafte Regierungskrisen, aber die Gefahr einer Ansteckung zum »dissent « ist vergleichsweise größer als innerhalb überschaubarerer Mehrheiten. Beispiele für die - auf den ersten Blick paradox erscheinenden - größeren Schwierigkeiten von Regierungschefs, mit größeren Mehrheiten zu agieren, finden sich in Deutschland wie in Großbritannien. ${ }^{46}$

$\mathrm{Zu}$ den ambivalentesten Faktoren, nicht nur im Zusammenhang mit politischer Führung, gehört der Faktor »Zeit«. Aus der Literatur über das politische Steuerungsvermögen von Ministern gegenüber der Ministerialverwaltung ist seit langem bekannt, dass der Einfluss politischer Spitzenakteure mit deren jeweiliger Amtsverweildauer zunimmt und oftmals erst nach einigen Jahren sein volles Ausmaß erreicht. ${ }^{47}$ Eine entsprechende Vermutung erscheint auch mit Blick auf die Durchsetzungsfähigkeit des Regierungschefs innerhalb der Exekutive gerechtfertigt, obwohl die Ressortfreiheit, die das Amt des Premierministers in der Moderne kennzeichnet, einem direkten Vergleich entgegensteht. Selbst dort, wo die Länge der Amtszeit nicht mit einem Zuwachs politischer Macht korreliert, bewahrt der Verzicht auf »term limits « parlamentarische Regierungschefs zumindest vor einem institutionell erzeugten »lame duck «-Status wie ihn amerikanische

44 Vgl. für die Bundesrepublik, Thomas Knoll, Das Bonner Bundeskanzleramt. Organisation und Funktionen 1949-1999, Wiesbaden 2004; Volker Busse, Bundeskanzleramt und Bundesregierung: Aufgaben - Organisation - Arbeitsweise, 4. Aufl., Heidelberg 2005.

45 So Nicole Bolleyer, »Minderheitsparlamentarismus - eine akteursorientierte Erweiterung der Parlamentarismus-Präsidentialismus-Typologie« in: Zeitschrift für Politikwissenschaft 11 (2001), S. 1519-1546, hier S. 1531. Zur jüngeren Geschichte des Minderheitsparlamentarismus in Dänemark und anderen Länder Skandinaviens gehört jedoch die Neigung von Regierungen, nach Möglichkeit vertragliche Vereinbarungen mit ausgewählten Oppositionsparteien zu treffen, die die Kalkulierbarkeit des parlamentarischen Verfahrens erhöhen sollen. Vgl. Flemming Juul Christiansen / Erik Damgaard, »Parliamentary Opposition under Minority Parliamentarism: Scandinavia « in: Ludger Helms (Hg.), Parliamentary Opposition in Old and New Democracies, London/New York 2009, S. 27-57.

46 Vgl. Helms, Presidents, Prime Ministers and Chancellors, aaO. (FN 4), S. 169 f., 199 f.

47 Richard Rose, The Problem of Party Government, London 1974. 
Präsidenten während der zweiten Hälfte ihrer zweiten Amtszeit erdulden müssen. ${ }^{48}$ Die parlamentarische Machtbasis regierender Mehrheiten ist dagegen üblicherweise einem Erosionsprozess ausgesetzt, der auch den politischen Status der Spitzenakteure der Exekutive nicht unberührt lässt. Dabei geht es weniger um Übertritte von Mitgliedern der Regierungsmehrheit zur Opposition, sondern um die »elektoralen Kosten« des Regierens. Vergleichende Studien belegen, dass sich die elektorale Unterstützungsbasis von Regierungen im Zuge mehrfacher Wiederwahlen in der großen Mehrzahl westeuropäischer Demokratien tendenziell verringert. ${ }^{49}$ In Systemen mit Nachwahlen (»by-elections «) während der Legislaturperiode sind auch diese weit überdurchschnittlich mit Mandatsverlusten der Regierungsparteien verbunden. ${ }^{50}$

$\mathrm{Zu}$ berücksichtigen sind ferner die massenmedialen Parameter eines Systems, die von der jüngeren Forschung zu Recht als genuin politische Komponenten demokratischer Regime betrachtet werden. ${ }^{51}$ Trotz unübersehbarer internationaler Konvergenztrends (vor allem mit Blick auf die Kommerzialisierung und strukturelle Fragmentierung von Mediensystemen) gibt es auch innerhalb der Familie der konsolidierten westlichen Demokratien nach wie vor signifikante Unterschiede in den medien- bzw. kommunikationsbezogenen Bedingungen politischer Führung. ${ }^{52}$ Am günstigsten beschaffen sind die medialen Parameter politischer Führung aus der Sicht von Regierungschefs bzw. Präsidenten in jenen Systemen, in denen der Fragmentierungsgrad der Medienlandschaft gering ist, in denen die öffentlich-rechtlichen Sender von der Mehrheitspartei kontrolliert werden und in denen der Anteil kommerzieller Medien klein (oder besser noch: nichtexistent), der journalistische Respekt vor den politischen Machthabern hingegen groß ist. Bis vor einigen Jahrzehnten entsprach von den großen alten Demokratien Westeuropas vor allem Frankreich in hohem Maße dieser aus Sicht der politischen Exekutive »idealen Konstellation «, und noch immer sind die medialen Bedingungen politischer Führung dort vergleichsweise günstig. ${ }^{53}$ Der internationale Entwicklungstrend auf diesem Gebiet zeigt jedoch eindeutig in Richtung einer strukturellen Erschwerung von

48 Mit Blick auf die Vereinigten Staaten deuten die Einsichten der jüngeren Forschung allerdings darauf hin, dass der Einfluss des 22. Amendments auf die Performanz unterschiedlicher Präsidenten alles in allem geringer ist als weithin angenommen. Vgl. David A. Crockett, »An Excess of Refinement<: Lame Duck Presidents in Constitutional and Historical Context « in: Presidential Studies Quarterly 38 (2008), S. 707-721.

49 Vgl. Wolfgang C. Müller / Kaare Strøm, »Conclusion: Coalition Governance in Western Europe« in: dies. (Hg.), Coalition Governments in Western Europe, Oxford 2000, S. 559-592, hier S. 589.

50 Vgl. David Sanders / Simon Price, »By-elections, Changing Fortunes, Uncertainty and the Midterm Blues«, in: Public Choice 95 (1998), S. 131-148.

51 Vgl. Michael Schudson, »The News Media as Political Institutions « in: Annual Review of Political Science 5 (2002), S. 249-269.

52 Vgl. Daniel C. Hallin / Paolo Mancini, Comparing Media Systems: Three Models of Media and Politics, Cambridge 2004.

53 Vgl. Peter Humphreys, Mass Media and Media Policy in Western Europe, Manchester/New York 1996, S. 111-158; Jean K. Chalaby, "French Political Communication in a Comparative Perspective: The Media and the Issue of Freedom« in: Modern and Contemporary France 13 (2005), S. 273-290.

ZfP 56. Jg. 4/2009 
»executive leadership«. Vor allem die kommerziellen Medien des audio-visuellen Sektors zwingen der Politik in beträchtlichem Maße ihre Funktionslogik auf und nötigen Regierungen zur Aufbietung spezieller finanzieller, personeller und zeitlicher Ressourcen für das »media management «, die möglicherweise an anderer Stelle fehlen. Eine weitere, noch immer unterschätzte restriktive Dimension der »Mediokratie « betrifft den signifikant erhöhten Zeitdruck, unter dem Regierungen agieren; von den restriktiven Effekten der kommerziellen Massenmedien auf die politischen Elitenrekrutierung ganz zu schweigen. ${ }^{54}$

Überwölbt werden die unterschiedlichen institutionellen und politischen Bedingungen politischer Führung durch die politisch-kulturellen Parameter eines Systems. Die Toleranz- bzw. Akzeptanzgrenzen von Gesellschaften in Bezug auf politisches Führungsverhalten und Entscheidungspolitik sind hochgradig verschieden. Diese bewusst zu machen und in den Dienst einer angemessenen Selbstreflexivität demokratischer Gesellschaften zu stellen, kann ebenfalls zu den Aufgaben einer gesellschafrelevanten »leadership«-Forschung gezählt werden. Die politisch-kulturellen Bedingungen eines Regimes müssen in jedem Fall gesondert studiert werden, denn nicht immer können gesellschaftliche Werthaltungen aus den bestehenden formalen institutionellen Arrangements herausgelesen werden. In vielen Ländern wird insbesondere das nach dem formalen Regelwerk mögliche Maß an majoritärer Regulierung von regierenden Mehrheiten nicht voll ausgeschöpft. Die häufige Bildung von Regierungskoalitionen, die nicht dem Kriterium der kleinstmöglichen Gewinnerkoalition (»minimum-winning «) entsprechen, ist nur der sichtbarste Ausdruck dessen. Allerdings ist davon auszugehen, dass es zu gezielten Aufweichungen des Mehrheitsprinzips selten ausschließlich mit Rücksicht auf spezifische Konsensbedürfnisse der Gesellschaft kommt. Eine eigenständige Rolle spielen die konkreten Interessen von Akteuren - etwa das Bestreben, Verantwortung für besonders schwierige Entscheidungen auf breitere Schultern zu verteilen - sowie stärker machtpolitische Aspekte, wie das Streben nach »Spaltung « des Oppositionslagers oder wahltaktische Erwägungen von Akteuren. ${ }^{55}$

Wie selbst dieser knappe Überblick erkennen lässt, ist es um die erkenntnisbezogene Leistungsfähigkeit der vergleichenden »leadership«-Forschung - trotz spezifischer Probleme - nicht schlecht bestellt. In vielen Bereichen ist der interessierte Betrachter nicht länger auf bloße Vermutungen über den Einfluss unterschiedlicher Faktoren angewiesen, sondern kann auf empirische Studien zurückgreifen, die sich zu einem Sittengemälde politischer Führung in der parlamentarischen Demokratie verdichten lassen.

$\mathrm{Zu}$ weit reichenden Einsichten in die Führungsleistung einzelner Amtsinhaber kann die »leadership«-Forschung insbesondere dann gelangen, wenn sie den internationalen Vergleich mit Gespür für die historische Dynamik der Bedingungen politischer Führung

54 Vgl. Ludger Helms, »Governing in the Media Age: The Impact of the Mass Media on Executive Leadership in Contemporary Democracies « in: Government and Opposition 43 (2008), S. 26-54.

55 Vgl. Wolfgang C. Müller, »Koalitionstheorien« in: Ludger Helms / Uwe Jun (Hg.), Politische Theorie und Regierungslebre. Eine Einführung in die politikwissenschaftliche Institutionenforschung, Frankfurt a.M./New York 2004, S. 267-301, hier S. 271 f. 
$\mathrm{zu}$ verbinden weiß. Einige der angesprochenen Faktoren (wie die parlamentarischen Mehrheitsverhältnisse) und weitere hier nicht weiter behandelte Aspekte (wie etwa die budgetären Spielräume von Regierungen) generieren auch innerbalb eines bestimmten Systems bzw. Regimes jeweils spezifische »Handlungskorridore« bzw. »Gelegenheitsfenster«. Ein empirischer Vergleich der konkreten Handlungsopportunitäten und -restriktionen erlaubt es, die Regierungen bzw. Regierungschefs eines Landes nach unterschiedlichen Gruppen zu ordnen, innerhalb derer »faire« Vergleiche politischer Führungsleistungen möglich sind. Als mustergültige Exemplifikation dieses Ansatzes im USamerikanischen Kontext kann die große Studie von William Lammers und Michael Genovese über die innenpolitischen Leistungen amerikanischer Administrationen von Truman bis Clinton gelten. ${ }^{56}$ In international vergleichenden Analysen ist es umso wichtiger, historische Unterschiede der Bedingungen politischer Führung innerhalb von Systemen $\mathrm{zu}$ »kontrollieren «, um Vergleiche mit womöglich doppelter Schieflage zu vermeiden. ${ }^{57}$

\section{Grenzen der vergleichenden »leadership«-Forschung}

Selbst historisch und international vergleichende Studien sehen sich jedoch spezifischen Problemen bzw. Grenzen gegenüber. Zu erwähnen sind zunächst Grenzen der empirischen Erforschung politischer Führung, für die nicht zuletzt der Umstand verantwortlich ist, dass der Regierungsbereich stärker als andere Sektoren politischer Systeme ein »Arkanbereich« geblieben ist. Ein nicht geringer Teil politischer Führungsleistung findet hinter verschlossen Türen statt und kann, wenn überhaupt, erst Jahrzehnte später auf der Basis mehr oder minder vollständiger Akten rekonstruiert werden. ${ }^{58}$ »Public leadership « mag, wie viele Autoren betonen, an Bedeutung gewonnen haben. Das Studium von Manifestationen öffentlicher bzw. öffentlichkeitsbezogener politischer Führung allein vermag aber freilich keine »ganzheitlichen« Bilder des politischen Führungs- bzw. Entscheidungsprozesses zu liefern. Das gilt umso mehr, als es zum erprobten Strategiearsenal von Regierungschefs (und anderen politischen Akteuren) gehört, für öffentliche Auftritte

56 Vgl. William Lammers / Michael Genovese, The Presidency and Domestic Policy, Washington, DC 2000.

57 Eine beeindruckende Demonstration kontextsensibler international vergleichender Analyse von »leadership «-Performance bieten David S. Bell / Erwin Hargrove / Kevin Theakston, »Skill in Context: A Comparison of Politicians « in: Presidential Studies Quarterly 29 (1999), S. 528-548. Als bedeutende weitere Leistung struktur- und kontextsensibler Zugänge kann gelten, dass sie dem problematischen "great men«-Ansatz der »leadership«-Forschung und seinem jüngeren, nicht minder problematischen Pendant, dem "great women «-approach den Boden entziehen. Vgl. zur Kritik beider Todd L. Pittinsky / Laura M. Bacon / Brian Welle, »The Great Women Theory of Leadership? « in: Barbara Kellerman / Deborah L. Rhode (Hg.), Women and Leadership. The State of Play and Strategies for Change, San Francisco 2007, S. 93-125.

58 Speziell in den USA wurden zuletzt sogar wachsende Probleme des Zugangs zu direkten Informationen konstatiert. Als möglicher Ausweg erscheinen Strategien des »elite oral history interviewing «, welche allerdings nicht frei von Problemen sind. Vgl. Russell L. Riley, »The White House as a Black Box: Oral History and the Problem of Evidence in Presidential Studies « in: Political Studies 57 (2008), S. 187-206. 
in bestimmte »Rollen « zu schlüpfen, ${ }^{59}$ von denen aus kaum zuverlässig auf deren Position im politischen Entscheidungsprozess geschlossen werden kann. Zum Teil könnte geradezu von einer gezielten strategisch motivierten »Vernebelung « von Entscheidungsprozessen gesprochen werden. Das international bekannteste Beispiel hierfür bleibt die viel beschriebene »hidden-hand presidency « Dwight D. Eisenhowers. ${ }^{60}$

Ansonsten stößt die politikwissenschaftliche »leadership«-Forschung insbesondere dort an ihre Grenzen, wo es um die Aufdeckung und Analyse der inneren Beweggründe des Führungsverhaltens von politischen Amtsinhabern geht. Das ist nicht nur den Schwierigkeiten der Entwicklung eines geeigneten analytisch-konzeptuellen Zugangs zu einem komplexen Gegenstand geschuldet. Entscheidender ist die mangelnde Sachkompetenz der Politikwissenschaft, das Innenleben von Persönlichkeiten zu analysieren und zu bewerten. Man wird diese Lücke als um so gravierender empfinden, je mehr man sich des beträchtlichen Potentials einschlägiger Studien bewusst ist, in denen tatsächlich psychologische oder psychoanalytische Ansätze mit politikwissenschaftlich relevanten Fragestellungen verknüpft werden. ${ }^{61}$

Als vielleicht wichtigster konzeptueller Beitrag psychologischer Arbeiten zur »leadership«-Forschung kann der Ansatz des »counterfactual thinking «, des »kontra-faktischen Denkens «, gelten. ${ }^{62}$ Er ist längst zu großem Einfluss auch außerhalb der politischen Psychologie gelangt. ${ }^{63}$ Sein Wert als wissenschaftliche Erkenntnisstrategie bleibt jedoch umstritten. In den Augen einiger Betrachter hat er die spekulative Tendenz des Studiums von politischer Führung eher noch verstärkt.

Angesichts dieser und anderer Schwierigkeiten kann es kaum verwundern, dass sich die »leadership «-Forschung ausgesprochen schwer damit tut, breiter dimensionierte empirische Theorien von »executive leadership « zu formulieren. In der jüngeren Forschung gibt es Versuche, die Anzahl berücksichtigter Faktoren zu minimieren, um auf diesem Wege das analytische Zugriffspotential theoriegeleiteten Studiums von politischer Füh-

59 Darauf wurde auch im deutschen Kontext bereits vor Jahrzehnten hingewiesen; vgl. Arnold J. Heidenheimer, »Der starke Regierungschef und das Parteien-System: Der >Kanzler-Effekt in der Bundesrepublik « in: Politische Vierteljabresschrift 1 (1961), S. 241-262, hier S. 250-251.

60 Vgl. Fred I. Greenstein, The Hidden-Hand Presidency: Eisenhower as Leader, New York 1982.

61 Die Pionierstudie des Forschungsbereichs stammt von James D. Barber, The Presidential Character: Predicting Performance in the White House, Englewood Cliffs, NJ 1977; einen Eindruck vom heutigen Stand der psychologischen Leadership-Forschung vermittelt die Studie von Jerrold M. Post, The Psychological Assessment of Political Leaders, Ann Arbor 2003; vgl. Ferner Joanne B. Ciulla (Hg.), Leadership at the Crossroads, Vol. 1: Leadership and Psychology, hrsg. von Crystal L. Hoyt / George R. Goethals / Donelson R. Forsyth, Westport, CT/London 2008.

62 Vgl. Fred I. Greenstein, »Can Personality and Politics Be Studied Systematically?« in: Political Psychology 13 (1992), S. 105-128.

63 Vgl. etwa Jeffrey M. Chwieroth, »Counterfactuals and the Study of the American Presidency« in: Presidential Studies Quarterly 32 (2002), S. 293-327; Duncan Brack / Iain Dale (Hg.), Prime Minister Portillo and Other Things That Never Happened: A Collection of Political Counterfactuals, London 2003. 
rung zu erhöhen. ${ }^{64}$ In dieselbe Richtung schlagen, bei allen Unterschieden, Ansätze aus dem Umfeld der Vetospielertheorie, die dem gewählten theoretischen Ansatz zuliebe von einer Statik des Verhältnisses im Verhältnis von »leader « und »leadership environment « ausgehen. ${ }^{65}$ In beiden Fällen sind die Vorteile des jeweils gewählten Zugangs greifbar, ohne dass ihr Preis - drohende Unterkomplexität und Realitätsferne - ganz zu leugnen wäre.

Im Gegensatz zu den soeben bezeichneten Grenzen der vergleichenden »leadership«Forschung ließen sich einige weitere Faktoren, die einem ungehinderten Erkenntniszuwachs im Wege stehen, ohne größeren Aufwand ausschalten. Das gilt insbesondere für Schwächen auf der terminologischen Ebene. Berühmt wurde Giovanni Sartoris Kritik an der - in der Tat irreführenden - Verwendung des Begriffs »coalition government « für den Zustand geteilter parteipolitischer Kontrolle von Kongress und Präsidentenamt in den USA. ${ }^{66}$ In Teilen der jüngeren Exekutivforschung wurde das Problem einer verfehlten Terminologie auf die Spitze getrieben: So zögern Paul Webb und Thomas Poguntke in ihrer viel beachteten Analyse über die vermeintliche »Präsidentialisierung « parlamentarischer Demokratien nicht, die Stärkung des Regierungschefs gegenüber dem Präsidenten eines Systems als eine Manifestation von »Präsidentialisierung « zu bezeichnen! ${ }^{67}$ Das mag im Rahmen der spezielleren konzeptuellen Parameter der betreffenden Studie gerechtfertigt erscheinen; dem Aufbau eines auch dem nicht Eingeweihten zugänglichen Wissensbestands über den Gegenstand kommt dies jedoch schwerlich zugute.

\section{Schlussbetrachtung: »Good governance« und der gesellschaftliche Stellenwert der vergleichenden $»$ leadership»-Forschung}

Die Einsicht in die maßgeblichen Strukturdeterminanten politischer Führung schafft die Voraussetzungen für eine Bewertung gezeigter »leadership«-Leistungen. Hinzu kommen muss jedoch die Verständigung auf taugliche Bewertungskriterien der politischen Führungsleistung von Regierungschefs, die ein gewisses Maß an Allgemeingültigkeit mit hinreichender Kontextsensibilität zu verbinden wissen.

Fliegauf, Kießling und Novy haben jüngst mit Verweis auf eine ältere Arbeit von Bert A. Rockman und Kent Weaver über den Charakter politischer Institutionen angeregt, bei der Bewertung der individuellen Führungsleistung von Regierungschefs zwischen »Effektivität « (bezogen auf das Ausmaß der Zielerreichung) und »Effizienz « (verstanden als der Quotient aus der Zielerreichung und den eingesetzten Mitteln) zu unterschei-

64 So mit Konzentration auf den Aspekt »leader/follower «, Mark T. Fliegauf / Andreas Kießling / Leonard Novy, "Leader and Follower - Grundzüge eines inter-personalen Ansatzes zur Analyse politischer Führungsleistung « in: Zeitschrift für Politikwissenschaft 18 (2008), S. 399-421.

65 Vgl. Stoiber, Politische Führung und Vetospieler, aaO. (FN 35), S. 39.

66 Giovanni Sartori, »Comparing and Miscomparing « in: Journal of Theoretical Politics 3 (1991), S. 243-257, hier S. $247 \mathrm{f}$.

67 Webb / Poguntke, »The Presidentialization of Contemporary Democratic Politics «, aaO. (FN 29), S. 343.

ZfP 56. Jg. 4/2009 
den. ${ }^{68}$ »Effizient ist politische Führung dann «, so die Autoren, »wenn die Zielvorstellungen des Führenden umgesetzt werden, ohne dass er dabei seine Macht einbüßt. « ${ }^{69}$ Ein solcher Effizienzbegriff macht Sinn, ist aber zugleich auf spezifische Weise verengt, da er allein auf die möglichen direkten Kosten für den betreffenden Amtsinhaber abhebt. Es ist nicht einzusehen, warum sich das Verständnis von »leadership«-Effizienz nicht zugleich auf das Ausmaß der Zielerreichung und den Einsatz anderer Ressourcen - wie insbesondere Zeit und Geld - erstrecken sollte.

Eher noch wichtiger erscheint eine zweite Erweiterung, die ebenfalls bereits unter anderem von Bert A. Rockman »vorgedacht « wurde: die Berücksichtigung von Aspekten, die mit Stichworten wie »Repräsentation«, »Integration « oder »Inklusion « umrissen werden können. ${ }^{70}$ Wie hoch Repräsentation und politische Integration als normative Leistungskriterien von politischer Führung im Verhältnis zu Effektivität und Effizienz bewertet werden, hängt maßgeblich von den politisch-kulturellen Parametern eines Gemeinwesens ab. Eine länderübergreifend »optimale« Lösung dieses komplexen Spannungsverhältnisses muss ein normatives Ideal bleiben; in der politischen Praxis demokratischer Regierungssysteme ist eine dauerhafte Überwindung dieses "great tradeoffs « ${ }^{71}$ demokratischer Politik nicht erreichbar. Gerade deshalb verdient die Entwicklung eines ausreichenden Gespürs für das gesellschaftlich jeweils Zumutbare zu den spezifischen Anforderungen erfolgreicher und legitimer (im Sinne von anerkennungswürdiger) politischer Führung in der Demokratie gerechnet zu werden.

Wie steht es aber schließlich um die Chancen der politikwissenschaftlichen »leadership«-Forschung, mit ihren Einsichten auch zu einer gesellschaftsrelevanten Ressource für das Gelingen der repräsentativen Demokratie zu werden? Zunächst muss freilich klar sein, dass von der Politikwissenschaft nicht erwartet werden kann, dass sie ihr Streben ausschließlich an ihrem potentiellen Einfluss auf die allgemeine Öffentlichkeit orientiert. Die heute international üblichen Kriterien zur Bewertung der Qualität wissenschaftlicher Forschung begünstigen in gewisser Weise sogar eher eine »Abkapselung « der Wissenschaft vom öffentlichen Diskurs über die Demokratie. Besonders hoch bewertet werden heute Beiträge in spezialisierten referierten Fachzeitschriften, mit Blick auf die selbst die darin vertretenen Autoren gelegentlich scherzen, dass sie selbst und die anonymen Gutachter in vielen Fällen die einzigen Leser ihres Beitrages seien. ${ }^{72}$ Obwohl die zentrale Bedeutung referierter Publikationen für die wissenschaftliche Qualitätssicherung außer Frage steht, ist die Gefahr nicht von der Hand zu weisen, dass die erstrebte leistungsbezogene Exklusivität hoch spezialisierter Fachzeitschriften begleitet wird von

68 Vgl. Fliegauf / Kießling / Novy, Leader and Follower, aaO. (FN 64), S. 410-415.

69 Ebd., S. 415.

70 Bei Rockman selbst dreht sich die Diskussion um die Begriffe bzw. Konzepte »representation « und "governance«; vgl. Bert A. Rockman, »Institutions, Democratic Stability and Performance « in: Metin Heper / Ali Kazancigil / Bert A. Rockman (Hg.), Institutions and Democratic Statecraft, Boulder, CO 1997, S. 11-34.

71 Vgl. Kenneth A. Shepsle, »Representation and Governance: The Great Legislative Trade-Off « in: Political Science Quarterly 103 (1988), S. 461-484.

72 Vgl. Robert Elgie, »Democratic Accountability and Central Bank Independence: A Reply to Various Critics« in: West European Politics 24 (2001), No.1, S. 217-221, hier S. 217. 
einer unerwünschten Exklusivität auf der Ebene der Distributionschancen entsprechender Veröffentlichungen.

$\mathrm{Zu}$ wünschen wäre deshalb eine größere Bereitschaft der Politikwissenschaft, stärker als »Kommunikator « in eigener Sache aufzutreten, womit ein spezieller Aufwand verbunden ist, der vom Wissenschaftssystem selbst zumeist wenig honoriert wird. Eine authentische Kommunikationsfunktion könnten dabei freilich am besten jene ausfüllen, die auch in der Forschung eine gewichtige Rolle spielen. In der Praxis ist es indes nicht selten so, dass schon die Abfassung von einführenden Lehrbüchern und erst recht von spezielleren Texten zur politischen Bildung gerne Vertretern »aus der zweiten Reihe« überlassen wird. Die Präsenz von Repräsentanten der politikwissenschaftlichen »Spitzenforschung « - in Deutschland institutionalisiert in Forschungsstätten wie dem Kölner MaxPlanck-Institut für Gesellschaftsforschung oder dem Wissenschaftszentrum Berlin für Sozialforschung - auf diesem Feld ist jedenfalls recht bescheiden.

Selbst im Falle einer wünschenswerten Intensivierung entsprechender Aktivitäten bliebe die politikwissenschaftliche »leadership «-Forschung freilich auf die Vermittlungsfähigkeit und -bereitschaft anderer Institutionen und Akteure angewiesen. Die in den meisten Ländern Westeuropas seit gut zwei Jahrzehnten zu beobachtende Herausbildung eines von kommerziellen Interessen dominierten Systems elektronischer Medien kommt einem breiten »Transfer « wissenschaftlicher Einsichten nicht gerade entgegen. Die Bereitschaft kommerzieller Medien, sich auf die Komplexität und Kontingenz demokratischer Politik ernsthaft einzulassen, bleibt aus nachvollziehbaren Gründen begrenzt. Ihre Präsenz beeinflusst zugleich die Programmstrukturen der öffentlich-rechtlichen Sender. ${ }^{73}$ Auch am staatlichen Engagement für politische Bildung, an der sich die Politikwissenschaft beteiligen könnte, ließe sich mit Blick auf viele Länder Kritik üben. ${ }^{74}$ Immerhin: Anders als in autoritären und totalitären Regimen darf die »leadership «-Forschung in der liberalen Demokratie zumindest darauf bauen, dass die im Fokus ihrer Analysen stehenden Akteure selbst einer Verbreitung ihrer Einsichten nicht prinzipiell im Wege stehen. Sofern es der politikwissenschaftlichen »leadership«-Forschung gelingt, nicht nur ihre eigenen Möglichkeiten und Grenzen, sondern auch jene von politischer Führung - von staatlicher Politik überhaupt - ins Licht zu rücken, sollte ihr Werk sogar im nachhaltigen Interesse von Regierungen und Regierenden liegen!

\section{Zusammenfassung}

Die Beschäftigung mit politischer Führung gehört seit einigen Jahren zu den Wachstumssektoren der internationalen Politikwissenschaft. Diese Entwicklung gibt Anlass, nach den Errungenschaften und Desideraten, nach den Möglichkeiten und Grenzen des

73 Vgl. Trine Syvertsen, »Challenges to Public Television in the Era of Convergence and Commercialization « in: Television and New Media 4 (2003), S. 155-175, hier S. 158-159.

74 Vgl. Olga Bombardelli, »Politische Bildung im europäischen Vergleich « in: Kurt Franke / Herbert Knepper (Hg.), Aufbruch zur Demokratie. Politische Bildung in den neunziger Jabren. Ziele, Bedingungen, Probleme, Opladen 1994, S. 83-99. 
Forschungsbereichs zu fragen. Der Schwerpunkt der Analyse liegt dabei auf dem Bereich von »executive leadership«, politischer Führung durch Regierungschefs. Besonders bewährt haben sich nach Auffassung des Verfassers Arbeiten, die in der Tradition eines »politisch erweiterten institutionellen Ansatzes « stehen. Probleme gibt es weiterhin nicht nur im Bereich der Theoriebildung, sondern auch auf dem Gebiet der empirischen Forschung. Zu den Anforderungen an eine zeitgemäße politikwissenschaftliche Beschäftigung mit »political leadership« muss neben wissenschaftsimmanenten Aspekten auch deren Beitrag zum politisch-gesellschaftlichen Diskurs außerhalb des akademischen Bereichs gezählt werden. Einige der offensichtlichsten Schwachpunkte in der Leistungsbilanz der Subdisziplin betreffen ihre eher geringe gesellschaftliche Ausstrahlung, ihren eher bescheidenen Beitrag zum Gelingen der repräsentativen Demokratie.

\section{Summary}

Fuelled by various dynamics in the world of politics, the study of political leadership has more recently turned into a growth sector of international political research. This development provides the starting point for the critical assessment of leadership studies offered in this paper, which focuses more specifically on the subject of executive leadership. Judged against possible alternatives, studies adopting a "politically expanded institutionalist « approach would appear to hold the greatest potential. However, serious problems persist not only at the level of theoretical generalizations, but also with regard to empirical analyses of executive leadership. Furthermore, reasonable expectations towards contemporary leadership studies tend to extend beyond the area of academic research and include other criteria such as the relevance of scholarship for society. It is in this area that contributions from political leadership research have remained remarkably modest.

Ludger Helms, Political Leadership in Liberal Democracies: Opportunities and Constraints of Comparative Research 